\title{
La Declaración universal de los derechos del animal
}

\section{Francisco J. Capacete González}

Abogado ejerciente especialista en Derecho Animal

Representante en Baleares del Proyecto Gran Simio

Recepción: Abril 2018

(c) (1)

Aceptación: Junio 2018

\section{Resumen}

En 1978 se presentó en público el primer texto de la Declaración Universal de los derechos del animal, promovida por la Liga Internacional de los Derechos de los Animales. Tras varios avatares y modificaciones se envió en varias ocasiones a la UNESCO para su aprobación. Sin embargo, esta no se ha materializado. Esta circunstancia no le resta validez ni importancia a la Declaración y muchos de los derechos recogidos en su texto ya forman parte de las legislaciones de diversos países.

Palabras clave: Derecho animal, declaración universal, derechos de los animales, UNESCO, ONU

\section{Abstract. The Universal Declaration of Animal Rights}

The first text of the Universal Declaration of animal rights, which was promoted by the International League of Animal Rights, was presented in public in 1978. After suffering several changes and modifications, it was sent on several occasions to the UNESCO for approval. However, the approval has not happened. This fact does not diminish neither the validity nor the importance of the Declaration and many of the rights included in its text are already part of the legislations of various countries.

Keywords: Animal Law, Universal Declaration, Animal Rights, UNESCO, ONU 
Innumerables son los medios y muchas las personalidades del ámbito jurídico y científico que citan esta Declaración, como la Fundación affinity y el partido animalista PACMA, en sus respectivas websites, apareciendo citado varias veces y mereciendo artículo propio en el libro EL DERECHO DE LOS ANIMALES (2015). También aparece citado en algunas disposiciones normativas vigentes, como la Ley 4/2016, de 22 de julio, de Protección de los Animales de Compañía de la Comunidad Autónoma de Madrid. Desde que en 1973 apareciera el texto inicial, redactado, entre otros, por el científico belga, Georges Heuse, Secretario General del Centro Internacional de Experimentación de Biología Humana de la UNESCO en París, se ha intentado equiparar la Declaración de los derechos de los animales a la Declaración universal de los derechos del hombre. Llevados más por la emotividad que por la realidad, una gran mayoría afirman que aquella Declaración ha sido aprobada por la UNESCO y por la ONU ${ }^{1}$. Debo reconocer que quien esto escribe también cometió el mismo error. La verdad es que ninguno de estos organismos ha mostrado su apoyo al texto. Veamos la historia de esta Declaración para resolver el malentendido.

Tras una campaña en el periódico "Le Parisien” y la cadena de radio RTL, se consiguieron dos millones de firmas apoyando la declaración inicial. Este apoyo les hizo pensar a los autores que era necesario dar una mayor difusión a la declaración y a tal efecto constituyeron la Liga Internacional de los Derechos de los Animales en 1976 en Ginebra. La presidencia fue confiada a Georges Heuse. Personalidades eminentes de las ciencias aportaron luego su contribución proponiendo modificaciones en el contenido y en la forma con el fin de aportar al texto más consistencia y una verdadera aportación científica. El texto modificado fue adoptado por la Liga Internacional de los Derechos del Animal en su $3^{\text {a }}$ Reunión celebrada en Londres del 21 al 23 de septiembre de 1977.

La primera presentación pública del texto tuvo lugar el 26 de enero de 1978 en el anfiteatro de la Universidad de Bruselas en presencia de más de 200 estudiantes y personalidades de la ciencia y de la política, entre ellos, un representante de la UNESCO, el Delegado de Togo, Presidente del Consejo para la Calidad de Vida. Georges Heuse defendía que esta Declaración tendría mayor eficacia en la protección de los animales si contara con el respaldo de una institución internacional. Pensó en la UNESCO, ya que el respeto por los derechos de los animales -pensaba Heuse- está directamente vinculado con la educación y ayudaba al fortalecimiento de la paz, dos de los objetivos de aquel organismo internacional.

La presentación oficial de la Declaración se fijó para el día 15 de octubre de 1978 en la gran sala de la Casa de la UNESCO en París. Fue leída en inglés, en árabe y en francés. La ceremonia se celebró delante un amplio auditorio que incluía a los embajadores de 14 países. Concluyó con la entrega de la Declaración al Director General de la UNESCO, Mr. Amadou-Mahtar M’Bow, para que fuera tratada en la Conferencia General de 1980. Georges Heuse veía este paso como el primer paso para que la Declaración llegara a ser adoptada por Organización de las Naciones Unidas antes de finales del siglo XX.

Desgraciadamente, a partir de la presentación oficial comenzaron a levantarse voces y opiniones en contra de la Declaración. Algunos sectores industriales veían en ella un grave peligro para sus intereses. Los científicos no terminaron de ponerse de acuerdo en la redacción. La polémica tuvo como consecuencia que no se tuviera en consideración ni por la UNESCO ni por la ONU.

Durante la Reunión de la Liga Internacional de los Derechos del Animal celebrada en junio de 1989 en Luxemburgo, se aprobó una redacción definitiva de la Declaración, con la intención de contar con el mayor número de adhesiones. El nuevo texto estaba compuesto de diez artículos en lugar de los catorce iniciales. Cuando fue terminada la redacción del

\footnotetext{
${ }^{1}$ Ver en http://www.filosofia.org/cod/c1977ani.htm, https://blog.pacma.es/2016/dia-internacional-de-los-derechos-de-los-animales/, https://www.animallaw.info/article/legislación-inglesa-y-norteamericana-derecho-animal-0 o el Decreto no 115/2015, de Protección de los Animales de la República de Honduras
} 
nuevo texto, la Liga francesa de los Derechos del Animal pidió a la UNESCO que recibiera una delegación con el fin de entregarlo, pero no fue posible. Ante la negativa de la UNESCO de tener en consideración la Declaración, fue enviada a los jefes de estado y a los magistrados más altos de los países representados en la Liga Internacional de los Derechos del Animal (Francia, Holanda, Alemania, Austria, Suiza, Bélgica, Noruega, Reino Unido, Portugal, España, Italia, Canadá, India, y Brasil). La realidad es que la Declaración Universal de los Derechos de los Animales fue leída y proclamada en la UNESCO-París y no por la UNESCO. La ONU tampoco la ha aprobado ni acogido nunca ${ }^{2}$.

Quienquiera que busque en internet el texto íntegro de la Declaración se encontrará con dos versiones, la de 1978 y la de 1989. Aquella compuesta de catorce artículos y ésta de diez. Ambas son igualmente válidas como referencia, pero ninguna tiene validez jurídica ni legal. Esto no significa que el esfuerzo realizado por sus promotores haya sido en vano. Algunos de los derechos recogidos en ambos textos ya forman parte de la legislación de muchos países.

Tomando como referencia el texto de 1978, vemos que los derechos recogidos en los artículos 2 a 4 (derecho al respeto, al cuidado, a la protección, prohibición de tratos crueles y derecho a una muerte indolora, así como a una vida en libertad para el caso de los animales salvajes), 6, 7, 8.b) y 14 (derecho a disfrutar de una longevidad natural en el caso de las mascotas, limitación del tiempo de trabajo, utilización de técnicas alternativas de experimentación animal, tutela de estos derechos por parte de la administración pública), están prácticamente integrados en la legislación española, a través de las leyes que protegen la biodiversidad y el patrimonio natural y el Código Penal que establece los delitos de maltrato y muerte de animales, además de las disposiciones autonómicas y municipales que protegen los animales que viven en el entorno humano. También tenemos que recordar que la normativa sobre sanidad animal establece que los conocimientos humanos deben ponerse al servicio de la salud de los animales.

La Ley 8/2003, de 24 de abril, de sanidad animal establece en su artículo 1 considera que los fines de esta disposición son, entre otros, la salud de los animales a través de la prevención y la mejora sanitaria. Las corporaciones locales están integrando a las entidades de protección animal en su esfera de acción, firmando convenios y creando foros de decisión conjunta, como la Mesa de Bienestar Animal del municipio de Sant Antoni de Portmany en Ibiza, o los convenios colaborativos de los municipios Cabra ${ }^{3}$, de Plasencia ${ }^{4}$, Yecla ${ }^{5}$, Alcaniz $^{6}$, entre otros, con entidades animalistas para proteger conjuntamente a los animales abandonados y callejeros. Y conviene recordar que España va a la zaga de otros países en cuanto a la protección de los derechos de los animales ${ }^{7}$. En Austria, la ley de 1 de julio de 1988 (BGB1 1988/179) introdujo el epígrafe 285a , que excluía a los animales de la consideración de cosas en propiedad. En Alemania, en 1990, se reformó la legislación civil mediante la Ley para la mejora de la situación jurídica del animal en el Derecho Civil, añadiendo en el capítulo 2, junto con las "Cosas”, otro apartado referido a los "Animales", para diferenciarlos de los meros objetos de propiedad. Más tarde, se modificó la constitución alemana (Grundgesetz, GG), elevando a rango constitucional la protección de los mismos junto a los fundamentos naturales de la vida. En Suiza hace más de treinta años que la ley protege a los animales con fuerza constitucional, incidiendo en la idea de la dignidad de las

\footnotetext{
${ }^{2}$ MOSTERÍN, J., Los derechos de los animales, en BALTASAR B., (Ed.), El Derecho de los Animales, (Madrid 2015) 61-62

${ }^{3}$ https://www.alcaniz.es/index.php?option=com content\&view=article\&id=764\%3Aconvenio-conasociacion-protectora-de-animales\&catid=166\%3Amedio-ambiente\&Itemid=808\&lang=es ${ }^{4}$ https://sede.plasencia.es/portal/transparencia/RecursosWeb/DOCUMENTOS/1/0 141_1.pdf 5http://www.yecla.es/Portals/0/Transpariencia/convenios/convenio_spandy_2017.pdf

${ }^{6}$ https://www.alcaniz.es/index.php?option=com content\&view=article\&id=764\%3Aconvenio-conasociacion-protectora-de-animales\&catid=166\%3Amedio-ambiente\&Itemid=808\&lang=es

${ }^{7}$ GIMÉNEZ CANDELA, T., Estatuto jurídico de los animales: aspectos comparados, en BALTASAR B., (Ed.), El Derecho de los Animales, (Madrid 2015) 149 y ss.
} 
criaturas vivas (artículo 80 Bundesvervassung). Algunos estados de EE.UU., como California, Illinois, Maine, Michigan y Oregon, han llevado al derecho positivo otros derechos incluidos en la Declaración Universal ${ }^{8}$.

La Declaración Universal de los Derechos de los Animales cada vez es más universal y cada vez menos una simple declaración de intenciones.

${ }^{8}$ https://www.animallaw.info/article/legislación-inglesa-y-norteamericana-derecho-animal-0 